\title{
Energetics and the Role of Defects in Fe(II)-Catalyzed Goethite Recrystallization from Multiscale Molecular Simulations
}

\author{
KeVIN M. Rosso ${ }^{1, *}$ AND PIOTR ZARZYCKI ${ }^{2}$ \\ ${ }^{1}$ Pacific Northwest National Laboratory, Washington, USA \\ ${ }^{2}$ Lawrence Berkeley National Laboratory, California, USA \\ *corresponding author: kevin.rosso@pnnl.gov
}

Goethite is one of the most stable and common iron (III) minerals at the Earth's near surface. However, recent isotopetracer studies suggest that goethite continuously recrystallizes in the presence of aqueous $\mathrm{Fe}$ (II) ions. Such studies often indicate the presence of two regimes of atom exchange kinetics, a rapid stage assigned to reactive defect sites initially available at particle surfaces, followed by slower continuous exchange. An autocatalytic solid-state electron conduction model coupling $\mathrm{Fe}(\mathrm{II})$ oxidative adsorption to its reductive release at spatially distinct sites has been proposed, but the thermodynamic driving force has yet to be pinpointed. Here, using a novel hybrid/reactive molecular simulation method, for goethite (110) surfaces at circumneutral $\mathrm{pH}$, we rigorously tested whether surface free energy minimization, including examining the role of structural defects, is sufficient to overcome the activation energy for interfacial electron transfer and solid-state electron conduction by polaronic hopping. The simulations quantitatively show that: i). On smooth stable surfaces at dynamic equilibrium the available room temperature thermal energy is sufficient to sustain the slow continuous regime of atom exchange kinetics via short intra-surface electron conduction pathways of 1-2 nm (3-5 Fe site hops); ii). In this slower regime, the model converges to atom exchange kinetics of $10^{-5} \mathrm{Fe} \mathrm{s}^{-1} \mathrm{~cm}^{-2}$, a rate also recently deduced from stochastic modeling of experimental data and linked to the reductive dissolution rate of goethite; iii). The driving force for initially rough defective goethite surfaces to smoothen can accelerate atom exchange to an extent quantitatively consistent with that observed in the initial rapid stage, in this case recruiting conduction pathways of up to 8 $\mathrm{nm}$. The findings suggest that the interaction of Fe(II) with initially defective goethite surfaces can drive, by the conduction model, atom exchange that is capable of recrystallizing the interiors of nanoscale particles, and that, closer to equilibrium on smooth surfaces, slower atom exchange continues in perpetuity but likely involving only the outermost atomic layers. 